\title{
Neurotrauma in the Time of SARS-COV 2: A Checklist for Its Safe Management
}

\author{
${ }^{1}$ Faculty of Medicine, Universidad Surcolombiana, Neiva, Colombia \\ ${ }^{2}$ Department of Neurosurgery and Gamma-Knife, All India Institute \\ of Medical Sciences, New Delhi, India \\ ${ }^{3}$ Department of Neurosurgery, National Institute of Mental Health \\ and Neurosciences, Bangalore, Karnataka, India \\ ${ }^{4}$ Department of Neurosurgery, All India Institute of Medical \\ Sciences, Bhopal, Madhya Pradesh, India
}

Luis Rafael Moscote Salazar ${ }^{1}$ Deepak Agrawal ${ }^{2} \quad$ Harsh Deora $^{3} \quad$ Amit Agrawal $^{4}$

\begin{abstract}
Address for correspondence Harsh Deora, MBBS, MS, MCh, DNB Department of Neurosurgery, National Institute of Mental Health and Neurosciences, Bangalore, Karnataka, India (e-mail: demo5601@gmail.com).
\end{abstract}

J Neurosci Rural Pract:2020;11:474-477

\begin{abstract}
Keywords

- neurotrauma

- COVID-19

- pandemic

- coronavirus

Neurotrauma is a critical public health problem that deserves the attention of the world health community. The unprecedented pandemic of SARS-COV 2 has led to a tremendous strain on medical facilities including intensive care and availability of blood products. In addition, due to lockdown in most nations and diverting of medical attention elsewhere, neurotrauma has taken a back seat. Despite this, any case of trauma presenting during this time should receive the best possible care. However, it is also imperative to safeguard the health care workers from this infection, too. The number of health care workers losing their lives to this infection is ever rising. We here present a possible workflow using a checklist approach such that errors and crossinfections are minimized and there is no reduction in the level of care received by any trauma case. This article has been written with a special focus on middle-income countries where resources may already be strained due to the sudden case burden. We hope to minimize death "caused" by COVID-19 and "related" to it.
\end{abstract}

\section{Introduction}

Traumatic brain injury not only represents a major cause of death in low- and middle-income countries but also in high-income countries. Additionally, it results in a significant number of people with disabilities, which implies a significant cost burden for health systems globally. It has been determined that after moderate and severe traumatic injury, brain tissue can be affected in more than $55 \%$ of cases. ${ }^{1-3}$ Humans are currently experiencing a pandemic period with the arrival of the SARS-CoV 2 (COVID 19) forcing neurosurgeons to rethink and redesign management strategies for their patients. During the pandemic, every patient should be suspected to be having SARS-CoV $2(+)$ to balance the safety of health care professionals and appropriate neurotrauma management of the patient(s).

\section{Triage}

With most countries and areas under lockdown, neurotrauma will not be at the forefront during this crisis but it does not imply that any case requiring emergent care receives a "less than ideal" treatment. The authors suggest that all patients must have blood drawn and a pooled sample sent for COVID 19 testing at regular intervals. Every health care professional who comes in contact with the patient should document their name on the cover sheet of the patient file so that an audit trail can be maintained. In case the pooled sample is COVID positive, individual testing of the pooled group, contact tracing, quarantining, and other measures should be put in place as per the local protocols. That said, we must be ready with a triage plan and simple two-point classification of potential trauma cases received during this time. 


\section{Requiring Urgent Surgery}

These are our utmost priority and present with conditions that are reversible, for example, extradural hematoma, herniating subdural, or acute spine injuries. Since many of these cases will present at centers remote from areas where operating facilities are available, patients that need the same must be identified and transported immediately to dedicated centers. Diffuse injuries can be managed at the local hospital/ center unless they require advanced monitoring. However, in a situation where critical care resources are extremely limited, the benefit of advanced monitoring is relatively limited.

\section{Injuries not Requiring Urgent Surgery}

Many injuries, both cranial and spinal, can be managed conservatively and the threshold for intervention changes with resources available. It is important, however, that the condition is still managed, and this may require joint working between local nonspecialists and neurotrauma care specialists requiring good communication. This may be by telephone or by telemedicine. For these reasons, as the situation with coronavirus escalates, being conversant with these technologies is important. Clinical decisions during a serious incident must consider the available facility for the current patient and also the impact this may have on the whole community.

\section{Protection of Surgeons and Staff}

\section{Preoperative Screening}

All incoming cases of trauma can be potentially corona positive and thus after a temperature check, a careful history query (especially the fever and cough manifestations in the last 2 weeks), and travel history must be noted by the doctor screening these cases. This screening doctor, if possible, should be conversant with clinical signs of herniation and deterioration such that he/she can differentiate cases based on urgency. Strict third-level protection (personal protective equipment) should be rigorously followed as numerous examples have been seen where one infected health care worker has led to a situation of mass quarantine with further burdening of health care services.

It may be advisable to do a nucleic acid sequencing of throat swab for preliminary diagnosis of COVID-19, although the results of the swab may be delayed after the surgery. In case any case is positive, the staff and surgeons may have to be quarantined immediately.

\section{Operative Room Preparation}

Division of the entire neurosurgical staff into multiple groups with one lead consultant, one senior resident, and one junior resident and operating in rotations would be an ideal scenario. We follow a policy of 1 week "on" and 2 weeks "off" so that there is mandatory quarantine after each week of duty. This ensures that only the team needs to be quarantined after any exposure and allows the rest of the system to work without problems. Pooled blood samples of all the patients in the department may be sent at least twice weekly to ensure that there COVID positive patients are detected early.
Negative suction operating theaters (OTs) may not be available at all centers and hence different OT complexes with wet fumigation after every case may be a more feasible alternative in such resource-limited settings. It goes without saying that all surgical clothes, surgical kit, and surgical shields should be disposable. Senior residents and trainees with a consultant on supervision should be preferred to shorten the operation duration. For complicated operations, two neurosurgeons can simultaneously work to reduce the duration of surgery. Double surgical gloves and minimal drilling/nibbling is recommended for neurosurgeons to avoid infection due to glove ruptures.

\section{Decompressive Craniectomy}

Decompressive craniectomy has been considered as one more tool in the treatment of elevated intracranial pressure that has not responded to medical treatment. ${ }^{4}$ Decompressive craniectomy surgery requires good planning and preparation and high standards of quality in terms of its implementation and perioperative management. Thus, the performing of strategies that enable the correct implementation of the process is welcome. A checklist in neurotrauma can be a tool made available to health care professionals to improve safety during surgery ( - Table $\mathbf{1}$ ).

Neurotrauma surgery can be enriched by the application of a strategy that ensures procedures as complex as a decompressive craniectomy. The checklist is aimed at an opportunity to verify and strengthen the requirements to perform a surgical procedure safely (i.e., decompressive craniectomy) and allow recommunication, foster teamwork between clinical disciplines, and identify the future requirements. ${ }^{1-3}$ This list is intended to be used in addition to the list prescribed by the World Health Organization, as a complement to it. We believe that the use of a simple additional checklist can help to carry out a decompressive craniectomy surgical procedure safely in patients with traumatic brain injury.

\section{Postsurgery Care}

Neurotrauma wards should be divided into several areas: patient rooms with infected cases and those with negative test results, while the nurse station and doctor should be where considered to be a clean area. Patient rooms were further divided into two partitions for suspected cases and confirmed cases. Individual accommodation would be ideally recommended for all patients but may not be possible in every situation and rigorous quarantine should be applied to the confirmed cases. Air conditioning should be switched off. Ideally, COVID positive patients should be shifted to a dedicated facility so that regular care in the department is not affected. Daily sterilization should be performed for every single room. Doctors and nurses must take strict third-level protection before entering patient rooms. Regular postoperative neuroimaging and laboratory examinations must be performed after hospitalization. 
Table 1 Neurotrauma surgical check list SARS-CoV 2

\begin{tabular}{|c|c|c|c|}
\hline & Item & Verified & Not verified \\
\hline 1 & Patient details & & \\
\hline 2 & Checking the indications & & \\
\hline 3 & Biochemical parameters including coagulation profile & & \\
\hline 4 & Imaging preparation & & \\
\hline 5 & Extent of craniectomy (hemicraniectomy/bilateral) & & \\
\hline 6 & Dural substitute/sealant material & & \\
\hline 7 & Anesthesia & & \\
\hline 8 & $\begin{array}{l}\text { Delimit caution areas } \\
\text { - Superior sagittal sinus } \\
\text { - Transverse sinus } \\
\text { - Venous lakes }\end{array}$ & & \\
\hline 9 & $\begin{array}{l}\text { Select head holder: } \\
\text { - Horseshoe } \\
\text { - Head fixation }\end{array}$ & & \\
\hline 10 & $\begin{array}{l}\text { Draw incision } \\
\text { - L.G. Kempe modified incision } \\
\text { - Large reverse question mark frontotemporoparietal incision } \\
\text { - Kjellberg type }\end{array}$ & & \\
\hline 11 & Asepsis and antisepsis & & \\
\hline 12 & Perform incision & & \\
\hline 13 & Scalp hemostasis & & \\
\hline 14 & Burr holes & & \\
\hline 15 & Paranasal sinus damage control & & \\
\hline 16 & Durotomy & & \\
\hline 17 & Dural hemostasis & & \\
\hline 18 & Inspect and correct & & \\
\hline 19 & Dural substitute & & \\
\hline 20 & Drainage & & \\
\hline 21 & $\begin{array}{l}\text { Bone flap } \\
\text { - Discard } \\
\text { - Abdominal subcutaneous pocket } \\
\text { - Cryopreservation }\end{array}$ & & \\
\hline
\end{tabular}

\section{Ventilatory Support}

Because of the dire shortage of ventilators, this resource should be used with utmost caution. There is a high chance of transmission in case any case requires emergency postoperative intubation, and hence if it is suspected that ventilation or respiratory support is expected, these cases can be shifted with a tube in situ or directly to the intensive care unit where personal protective equipment would be sufficient. The air ducts of the ventilator should be replaced daily. Nutrition support is often a neglected area but is as important for maintaining immunological function and reducing the possibility of virus and bacterial infection. If the pulmonary computed tomography scan and nucleic acid sequencing of throat swab are negative for COVID-19 after 2 weeks, the quarantine could be terminated and patients transferred from rooms of suspected cases. The recovered patients without COVID-19 should be transferred to the neurosurgery recovery ward located on another floor or to the rehabilitation center directly.

Perhaps one of the best examples is from the largest neurosurgical centers of Wuhan, China, where surgical theaters and care were operational during this time with a claimed "zero infection" protocol. ${ }^{5}$ 


\section{Conclusion}

Great challenges lead to even greater results. As neurosurgeons, we have always been ahead of the curve. Till there is definite treatment or vaccine for coronavirus the threat looms large. As always, any prevented neurotrauma trumps a treated one. The public should be educated to be cautious in preventing and controlling this disease. Otherwise, the mortality "related" to coronavirus shall be far greater than that "caused" by it.

\section{Funding}

None.

\section{Conflict of Interest}

None declared.

\section{References}

1 de Vries EN, Eikens-Jansen MP, Hamersma AM, Smorenburg SM, Gouma DJ, Boermeester MA. Prevention of surgical malpractice claims by use of a surgical safety checklist. Ann Surg 2011;253(3):624-628

2 Chua C, Wisniewski T, Ramos A, Schlepp M, Fildes JJ, Kuhls DA. Multidisciplinary trauma intensive care unit checklist: impact on infection rates. J Trauma Nurs 2010;17(3):163-166

3 de Vries EN, Hollmann MW, Smorenburg SM, Gouma DJ, Boermeester MA. Development and validation of the SURgical PAtient Safety System (SURPASS) checklist. Qual Saf Health Care 2009;18(2):121-126

4 Honeybul S, Decompressive craniectomy for severe traumatic brain injury: a review of its current status. J Neurol Neurophysiol. 2013;04(03).

5 Tan YT, Wang JW, Zhao K, et al. Preliminary recommendations for surgical practice of neurosurgery department in the central epidemic area of 2019 coronavirus infection. Curr Med Sci 2020;40(2):281-284 